\title{
Desenlaces maternos, fetales y perinatales de las gestantes con diabetes en manejo con bomba de insulina en el Hospital Universitario San Ignacio
}

\author{
Jaime Silva $H^{1}{ }^{1}$, Sandra Buitrago $F^{1}{ }^{1}$, Ana Gómez $M{ }^{2}{ }^{2}$, Lisseth Marín $C .^{2}$ \\ ${ }^{1}$ Departamento de Ginecología y Obstetricia, ${ }^{2}$ Unidad de Endocrinología. Hospital Universitario San Ignacio, Facultad de \\ Medicina, Pontificia Universidad Javeriana. Bogotá, Colombia.
}

\section{RESUMEN}

Antecedentes: La diabetes en el embarazo se asocia a un incremento considerable en la morbimortalidad materna, fetal y perinatal. Objetivo: Determinar los desenlaces maternos, fetales y perinatales de las gestantes con diagnóstico de diabetes pre gestacional en manejo con bomba de insulina en el Hospital Universitario San Ignacio. Método: Estudio de corte transversal en 24 gestantes con diagnóstico de diabetes en manejo con bomba de insulina quienes asistieron a control por Endocrinología y Alto Riesgo Obstétrico en el Hospital Universitario San Ignacio, entre Septiembre de 2009 y Diciembre de 2015. Resultados: La complicación materna más frecuente fue la hipoglicemia sintomática $(50 \%)$, en segundo lugar los trastornos hipertensivos asociados al embarazo (45,8\%). Las complicaciones neonatales más frecuentes fueron el síndrome de dificultad respiratoria del recién nacido $(37,5 \%)$ y la hiperbilirrubinemia $(37,5 \%)$. La hemoglobina glicosilada mayor del $6 \%$ se relacionó con el $71 \%$ de pacientes con macrosomía fetal. Conclusiones: La bomba de infusión de insulina representa una estrategia relativamente nueva dentro de los protocolos de manejo de diabetes pre gestacional y embarazo. Representaría una opción de manejo segura y fácil para el control de la glicemia en pacientes con indicaciones precisas.

PALABRAS CLAVE: Diabetes, complicaciones perinatales, bomba de infusión de insulina

\section{SUMMARY}

Background: Diabetes in pregnancy is associated to a considerable increase in the maternal, fetal and perinatal morbidity and mortality. Objective: Determine the maternal, fetal and perinatal outcomes of patients with pre gestational diabetes being treated with insulin pump at Hospital San Ignacio. Methods: Cross-sectional study of 24 patients diagnosed with diabetes being treated with insulin pump who assisted to controls made by Endocrinology and Obstetrical High-Risk units at Hospital San Ignacio from September 2009 to December 2015. Results: The most common maternal complication was symptomatic hypoglycemia (50\%) followed by hypertensive disorders of pregnancy (45.8\%). Most common neonatal complications were respiratory distress syndrome (37.5\%) and hyperbilirubinemia (37.5\%). Glycosylated hemoglobin higher than $6 \%$ was related with $71 \%$ of patients who developed fetal macrosomia. Conclusions: The insulin infusion pump is a relatively new strategy management of pre gestational diabetes and pregnancy. Representing a safe and easy option for glycemic control in patients with precise indications.

\section{KEY WORDS: Diabetes, perinatal complications, insulin infusion pump}




\section{INTRODUCCIÓN}

Según la OMS la Diabetes Mellitus representa el tercer problema de salud pública más importante del mundo. Se calcula que en el 2014 la prevalencia mundial fue del $9 \%$ (1) y para el 2030 será la séptima causa de mortalidad (2). La diabetes gestacional se define como la hiperglicemia que se detecta por primera vez durante el embarazo y alcanza una prevalencia hasta del $14 \%(1,3)$. Tanto la diabetes gestacional como la diabetes pregestacional se asocian a un incremento en la morbimortalidad materna, fetal y perinatal.

En el Hospital San Ignacio manejamos gestantes con diabetes pregestacional en forma interdisciplinaria con la unidad de endocrinología. En las pacientes en quienes es difícil una adecuada titulación de la dosis de insulina, existe la opción de instaurar un sistema de infusión continua de insulina mediante un dispositivo tipo bomba integrado a monitoreo continuo de glucosa. Este dispositivo ha sido propuesto como una opción segura y efectiva para el control metabólico de éstas pacientes, permitiendo un estilo de vida más flexible.

El objetivo del presente estudio es conocer los desenlaces maternos fetales y perinatales de las pacientes en manejo con bomba de insulina en el Hospital San Ignacio.

\section{PACIENTES Y MÉTODOS}

Estudio de tipo descriptivo, incluyendo 24 gestantes con diagnóstico de diabetes que cumplían criterios para manejo con bomba de insulina (mal control metabólico o hipoglicemias inadvertidas o severas) y asistían a controles por Endocrinología y Alto Riesgo Obstétrico en el Hospital San Ignacio, Bogotá, Colombia, entre Septiembre de 2009 y Diciembre de 2015.

La bomba de infusión de insulina es un dispositivo portátil constituido por un reservorio de insulina y un catéter de administración que se inserta en el tejido subcutáneo del abdomen. Permite programar la administración de insulina de acción rápida las 24 horas del día (infusión basal) y previo a la ingesta de alimentos (bolos). La administración de bolos preprandiales debe ser realizada por el paciente y dependerá del número de carbohidratos ingeridos.

El monitoreo continuo de glucosa funciona mediante la instalación de electrodos subcutáneos que miden la concentración de glucosa intersticial y reportan el promedio de estos niveles cada 5 minutos a nivel de la dermis. El tiempo de vida útil de los electrodos es de 2 a 6 días y requieren calibración con la glucemia capilar por lo cual sigue siendo indispensable el auto monitoreo. Este sistema aporta información muy valiosa acerca del comportamiento de la glucemia diurno, nocturno, preprandial, postprandial y en circunstancias especiales como ejercicio, estrés, sueño, infección, entre otros. Además, otorga un beneficio potencial en disminuir la frecuencia y/o la intensidad de las hipoglicemias, ya que es el único método que permite programar el descenso de la infusión de insulina basal que administra la bomba, en los momentos en que se identifica que el paciente tiene cotidianamente mayor riesgo de hipoglicemias.

Se utilizaron bombas de insulina integradas a monitoreo continuo de glucosa marca Medtronic, modelos 722 y 254 Veo. Este último modelo con función de suspensión automática en caso de hipoglicemia. Los tipos de insulina administradas fueron insulinas análogas de corta acción lispro y aspart.

Se organizó una base de datos a partir de la revisión de las historias clínicas, diligenciando un instrumento de recolección de datos, el cual se analizó mediante la construcción de tablas dinámicas en Excel 2013.

Esta investigación fue autorizada por el Comité de Ética e Investigación del Hospital San Ignacio.

\section{RESULTADOS}

Se incluyeron un total de 24 pacientes cuyo control se realizó en el Hospital Universitario San Ignacio. Las variables demográficas se describen en la Tabla I.

De las 24 pacientes estudiadas $22(91,6 \%)$ fueron llevadas a cesárea. La indicación más frecuente fue el antecedente de cesárea (25\%) y en segundo lugar el estado fetal no satisfactorio $(16,6 \%)$. Dos pacientes tuvieron partos vaginales eutócicos sin complicaciones y no se reportaron traumas obstétricos asociados.

Se reportaron 7 casos $(29,1 \%)$ de peso neonatal $\geq 3800 \mathrm{~g}$. Del grupo de pacientes que presento macrosomía fetal el $57 \%$ presentó síndrome de dificultad respiratoria y el $42,8 \%$ hipoglicemia neonatal. Se destaca que la paciente con el mayor peso neonatal presento síndrome de dificultad respiratoria y adicionalmente tuvo un pobre control diabético.

La indicación más frecuente para el inicio de bomba de insulina fue la presencia de mal control metabólico $(91,6 \%)$ y en segundo lugar la presencia de hipoglicemias inadvertidas o severas $(87,5 \%)$. Sin embargo, es importante resaltar que en la mayoría de los casos existió más de una indicación para el inicio de la bomba de insulina $(79 \%)$.

De las 24 pacientes incluidas en el estudio el $66,6 \%(16 / 24)$ se encontraban en manejo con bomba de insulina antes del embarazo. Las 8 pacientes restantes iniciaron manejo con bomba de insulina entre el I y el II trimestre como se muestra en la Tabla I. 
Tabla I

EMBARAZADAS DIABÉTICAS EN MANEJO CON BOMBA DE INSULINA, HOSPITAL SAN IGNACIO

\begin{tabular}{cccccccccc}
\hline Pacientes & Gestaciones & $\begin{array}{c}\text { Parto } \\
\text { vaginal }\end{array}$ & Cesárea & Aborto & $\begin{array}{c}\text { Vía de } \\
\text { parto }\end{array}$ & $\begin{array}{c}\text { Indicación de } \\
\text { intervención }\end{array}$ & $\begin{array}{c}\text { Indicación } \\
\text { bomba de } \\
\text { insulina }\end{array}$ & $\begin{array}{c}\text { EG inicio } \\
\text { bomba } \\
\text { insulina }\end{array}$ & $\begin{array}{c}\text { Peso } \\
\text { RN (g) }\end{array}$ \\
\hline 1 & 1 & 1 & 0 & 0 & Vaginal & N/A & 1 & $0^{*}$ & 2395 \\
2 & 1 & 0 & 1 & 0 & Cesárea & DDD & 1 & $0^{*}$ & 3990 \\
3 & 1 & 0 & 1 & 0 & Cesárea & DCP & 1 & $0^{*}$ & 3875 \\
4 & 1 & 0 & 1 & 0 & Cesárea & DCP & 1 & $0^{*}$ & 3850 \\
5 & 3 & 0 & 1 & 2 & Cesárea & PEG & 1 & $0^{*}$ & 2710 \\
6 & 1 & 0 & 1 & 0 & Cesárea & EFNS & 1 & $0^{*}$ & 4040 \\
7 & 1 & 0 & 1 & 0 & Cesárea & MCS & 1 & 17 & 3260 \\
8 & 1 & 0 & 1 & 0 & Cesárea & SFA & 1 & 16 & 3460 \\
9 & 1 & 0 & 1 & 0 & Cesárea & EFNS & 1 & 20 & 3805 \\
10 & 1 & 0 & 1 & 0 & Cesárea & EFNS & 1 & 21 & 2860 \\
11 & 2 & 0 & 2 & 0 & Cesárea & ANT & 1 & $0^{*}$ & 3790 \\
12 & 2 & 0 & 2 & 0 & Cesárea & ANT & 1 & $0^{*}$ & 3970 \\
13 & 4 & 0 & 3 & 1 & Cesárea & ITA & 1 & 14 & 4855 \\
14 & 3 & 0 & 2 & 1 & Cesárea & ANT & 1 & 26 & 2585 \\
15 & 3 & 2 & 1 & 0 & Cesárea & PMT & 1 & $0^{*}$ & 1439 \\
16 & 1 & 0 & 1 & 0 & Cesárea & EM & 1 & $0^{*}$ & 2845 \\
17 & 1 & 0 & 1 & 0 & Cesárea & SFA & 1 & 12 & 2285 \\
18 & 3 & 0 & 2 & 1 & Cesárea & CNAPI & 2 & $0^{*}$ & 2390 \\
19 & 3 & 0 & 2 & 1 & Cesárea & ANT & 2 & $0^{*}$ & 3170 \\
20 & 2 & 0 & 2 & 0 & Cesárea & ANT & 1 & $0^{*}$ & 2785 \\
21 & 2 & 1 & 0 & 1 & Vaginal & N/A & 1 & $0^{*}$ & 3625 \\
22 & 2 & 0 & 1 & 1 & Cesárea & PEG & 1 & $0^{*}$ & 1450 \\
23 & 2 & 0 & 2 & 0 & Cesárea & ANT & 1 & 23 & 2595 \\
24 & 2 & 1 & 1 & 0 & Cesárea & EFNS & 1 & $0^{*}$ & 1980 \\
\hline
\end{tabular}

N/A: No Aplica; RN: Recién nacido; EG: Edad gestacional. DDD: Detención en la dilatación y el descenso; DCP: Desproporción cefalopélvica; PEG: Preeclampsia grave; MCS: Sospecha de macrosomía; SFA: Sufrimiento fetal agudo; EFNS: Estado fetal no satisfactorio; ANT: Antecedente de cesárea; ITA: Iterativa; PMT: Prematurez; EM: Edad materna; CNAPI: Condiciones no aptas para inducción. Indicaciones para inicio de bomba de insulina: 1=Mal control metabólico, $2=$ Hipoglicemias severas o inadvertidas. $0^{*}$ : Inicio de bomba de insulina pregestacional.

El $41,7 \%(10 / 24)$ de nuestras pacientes tuvieron $<37$ semanas, el $16,7 \% \leq 34$ semanas $(4 / 24)$ y el $25 \%>34$ semanas $(6 / 24)$. Tres ingresaron con parto pretérmino en fase avanzada.

Las 7 pacientes restantes fueron llevadas a cesárea antes del término por estado fetal no satisfactorio/sufrimiento fetal agudo ( $30 \%$ de los casos), o trastorno hipertensivo del embarazo (30\% de los casos) y solo una paciente fue llevada a cesárea por inicio de trabajo de parto regular a la semana 36 con antecedente de cesárea.

En la Tabla II se muestran las complicaciones perinatales. Las más frecuentes fueron el síndrome de dificultad respiratoria del recién nacido $(37,5 \%$ de los casos), de estos el $44 \%$ se encontraban a término al nacer. La hiperbilirrubinemia también se presentó en el $37,5 \%$ de los casos. La mayoría $(79,1 \%)$ de los recién nacidos presentaron complicaciones y $20,9 \%$ de los recién nacidos no tuvieron ninguna complicación ni requirieron hospitalización en unidad de cuidado intensivo. El $25 \%$ de los recién nacidos presentaron bajo peso al nacer. No se presentaron recién nacidos con retardo en el crecimiento intrauterino. 


\section{Tabla II COMPLICACIONES PERINATALES}

\begin{tabular}{lr}
\hline Complicaciones perinatales & \multicolumn{1}{c}{$\mathrm{n} / \mathrm{N}(\%)$} \\
\hline Bajo peso al nacer & $6 / 24(25)$ \\
Hiperbilirrubinemia & $9 / 24(37,5)$ \\
Hipoglicemia & $7 / 24(29,2)$ \\
Enterocolitis necrotizante & $0 / 24(0,0)$ \\
Macrosomía & $7 / 24(29,2)$ \\
Síndrome de dificultad respiratoria del & $9 / 24(37,5)$ \\
recién nacido & \\
\hline
\end{tabular}

La Tabla III presenta las complicaciones maternas. La complicación más frecuente fue la hipoglicemia sintomática $(50 \%)$ seguida de los trastornos hipertensivos asociados al embarazo: preeclampsia con criterios de severidad (25\%) e hipertensión gestacional $(16,6 \%)$. Del total de pacientes sólo 2 requirieron hospitalización en Unidad de Cuidado Intensivo relacionado con el desarrollo de preeclampsia con criterios de severidad y Síndrome Hellp.

De las 7 pacientes que desarrollaron macrosomía fetal el $85 \%$ tuvieron hemoglobinas glicosiladas mayores a $6 \%$ en el segundo y tercer trimestre.

Se presentó solo una malformación fetal representada por un caso de microtia en una paciente con diabetes pregestacional, que inicio su manejo con bomba de insulina a las 13 semanas de embarazo y cuya hemoglobina glicosilada pregestacional se cuantificó en 9,6\%.

\section{DISCUSIÓN}

La diabetes pregestacional es una entidad que ocurre en el 8-14\% de las pacientes y tiene consecuencias maternas y fetales importantes. Se calcu-

\section{COMPLICACIONES MATERNAS}

\begin{tabular}{lr}
\hline Complicaciones maternas & \multicolumn{1}{c}{$\mathrm{n} / \mathrm{N}(\%)$} \\
\hline Cetoacidosis diabética & $2 / 24(8,3)$ \\
Hipertensión gestacional & $4 / 24(16,7)$ \\
Hipoglicemia sintomática & $12 / 24(50,0)$ \\
Preeclampsia sin criterios de severidad & $1 / 24(4,2)$ \\
Preeclampsia con criterios de severidad & $6 / 24(25,0)$ \\
Síndrome Hellp & $1 / 24(4,2)$
\end{tabular}

la que el $47 \%$ de los recién nacidos hijos de madres diabéticas ingresan a unidad de cuidado intensivo neonatal por síndrome de dificultad respiratoria, prematurez e hipoglicemia entre otros (4). Las madres en un alto porcentaje desarrollan hipoglicemia y hasta $3 \%$ presentan cetoácidosis diabética (5).

Uno de los grandes retos para evitar estas complicaciones es el control glicémico, para esto se cuenta con dos esquemas de aplicación de insulina: uno de ellos es la administración de múltiples dosis combinando los diferentes tipos de insulina y el otro es la administración a través de bombas de infusión.

La evidencia respecto a los beneficios de las bombas de insulina en comparación con la aplicación de múltiples dosis en relación con los desenlaces maternos y perinatales es insuficiente. Sin embargo el uso de bombas de infusión continua de insulina se ha planteado en las pacientes que presentan mal control metabólico e hipoglicemias inadvertidas o severas.

Los resultados de éste estudio arrojaron un alto porcentaje de cesárea $(91,6 \%)$ sin embargo la indicación de la intervención del parto no estuvo relacionada con la patología de base sino con indicaciones obstétricas diferentes. Las pacientes con macrosomía fetal $(29,1 \%)$ tuvieron un peor desenlace perinatal presentando mayor frecuencia de síndrome de dificultad respiratoria e hipoglicemia neonatal, incluso en los recién nacidos a término. Esto se correlacionó con mayores valores de hemoglobina glicosilada a lo largo del embarazo, hallazgos que se corresponden con la literatura en donde el resultado perinatal va de la mano con un adecuado control glicémico (6).

Los recién nacidos de madres diabéticas tiene mayor riesgo de desarrollar síndrome de dificultad respiratoria como resultado del hiperinsulinismo fetal que altera la síntesis de surfactante pulmonar incluso después de las 34 semanas de gestación (7). En un estudio realizado por Moore (8), en el que se incluyeron 295 pacientes diabéticas y 590 controles, se encontró que en las pacientes diabéticas la maduración pulmonar fetal está retrasada entre 1 y 1,5 semanas. Estos resultados se relacionan con lo encontrado en el presente estudio, en el que una de las complicaciones más frecuentes fue el Síndrome de dificultad respiratoria del recién nacido representando el $37,5 \%$ de los casos, de los cuales el $44 \%$ se encontraban a término al nacer. Dentro de los desenlaces perinatales la hiperbilirrubinemia constituye una morbilidad importante ocurriendo en el estudio en el $37 \%$ de las pacientes estudiadas, superando la hipoglicemia neonatal.

Los desenlaces maternos más frecuentes son la hipoglicemia materna y los trastornos hipertensivos asociados al embarazo. En este estudio se encontró en el $50 \%$ y el $45,8 \%$ respectivamente. 
En la literatura existen muy pocos estudios que evalúen las indicaciones para el uso de bombas de infusión de insulina en el embarazo y esto ha hecho necesario extrapolar información de estudios realizados en población no embarazada. En el 2015 la Asociación Americana de Endocrinología Clínica consideró apropiado el uso de bombas de insulina en pacientes con Diabetes Mellitus tipo 1 o 2 en quienes no se ha logrado un adecuado control a pesar de la aplicación de múltiples dosis de insulina (9). En el Hospital San Ignacio las indicaciones para inicio de bomba de insulina en el embarazo incluyen mal control metabólico, hipoglicemias inadvertidas o frecuentes. En las pacientes estudiadas la indicación más frecuente fue la presencia de hipoglicemias inadvertidas (87\%) y en segundo lugar el mal control metabólico (83\%). La mayoría de las pacientes con hipoglicemias inadvertidas presentaban también mal control metabólico.

En relación a la hemoglobina glicosilada se encontraron los siguientes hallazgos; el $87 \%$ de las pacientes que ingresaron a bomba de insulina durante el embarazo tenían valores de hemoglobina glicosilada alterados y al finalizar el embarazo el $57 \%$ de ellas tenían hemoglobinas glicosiladas en rangos normales. El 13\% de las pacientes restantes ingresaron a bomba por otras razones diferentes.

El promedio de hemoglobina glicosilada agrupando todas las pacientes en manejo con bomba de insulina durante el embarazo fue de $7,7 \%$ y $6,6 \%$ en el I y III trimestre respectivamente; resultados similares a los encontrados en un estudio multicéntrico observacional recientemente publicado en el que se demostró que el uso de bombas de insulina en el embarazo se asocia a niveles menores de hemoglobina glicosilada $(6,9 \%$ y $6,4 \%$ en I y III trimestre respectivamente) (10).

Es importante destacar que este estudio es de tipo retrospectivo e idealmente ésta patología debería evaluarse con estudios de casos y controles ya que la diabetes pregestacional plantea muchas variables que deben ser tenidas en cuenta en dos grupos diferentes de evaluación (tiempo de evolución de la patología, adherencia al manejo, comorbilidades asociadas, respuesta clínica a la insulina entre otros). La bomba de infusión de insulina representaría una opción de manejo en pacientes diabéticas pregestacionales con las indicaciones anotadas anteriormente.

\section{CONCLUSIÓN}

La bomba de infusión de insulina es una estrategia relativamente nueva dentro de los protocolos de manejo de diabetes pregestacional y embarazo. Representaría una opción de manejo segura y fácil para el control de la glicemia en pacientes con indicaciones precisas.

\section{REFERENCIAS}

1. Global status report on noncommunicable diseases 2014. Geneva, World Health Organization, 2012. Disponible en: http://apps.who.int/iris/bitstre am/10665/148114/1/9789241564854_eng.pdf. Acceso el $03 / 07 / 2016$.

2. Mathers CD, Loncar D. Projections of global mortality and burden of disease from 2002 to 2030 . PLoS Med 2006;3(11):e442-e442.

3. American Diabetes Association. Diagnosis and classification of diabetes mellitus. Diabetes Care 2014;37:S81-S90.

4. Hay WW Jr. Care of the infant of the diabetic mother. Cur Diab Rep 2012;12(1):4-15

5. Sibai BM, Viteri OA. Diabetic ketoacidosis in pregnancy. Obstet Gynecol 2014;123(1):167-78.

6. The HAPO Study Cooperative Research Group. Hyperglycemia and adverse pregnancy outcomes. N Engl J Med 2008;358(19):1991-2002.

7. Mitanchez D, Yzydorczyk C, Siddeek B, Boubred F, Benahmed M, Simeoni U. The offspring of the diabetic mother - short- and long-term implications. Best Pract Res Clin Obstet Gynaecol 2015;29(2):256-69.

8. Moore TR. A comparison of amniotic fluid fetal pulmonary phospholipids in normal and diabetic pregnancy. Am J Obstet Gynecol 2002;186(4):641-50.

9. Handelsman $Y$, Bloomgarden ZT, Grunberger G, Umpierrez G, Zimmerman RS, Bailey TS, et al. American Association of Clinical Endocrinologists and American College of Endocrinology - clinical practice guidelines for developing a diabetes mellitus comprehensive care plan - 2015. Endocr Pract 2015;21Suppl 1:1-87.

10. Kallas-Koeman M, Kong JM, Klinke JA, Butalia S, Lodha AK, Lim KI, Duan QM, Donovan LE. Insulin pump use in pregnancy is associated with lower $\mathrm{HbA} 1 \mathrm{c}$ without increasing the rate of severe hypoglycaemia or diabetic ketoacidosis in women with type 1 diabetes. Diabetologia 2014;57(4):681-9. 